$\xi=-1$

\title{
Synthesis of Linear Array Antenna for Required Range of Beam Width Using Optimization Algorithms
}

\author{
N. Venkateswara rao*, G. Challa Ram \\ Department of electronics and communication engineering, SRKR Engineering college, Bhimavaram, Andhra Pradesh -534204. \\ *Corresponding author E-mail: vrnagalla @ gmail.com; Tel: +91-9490031988; Fax:+ 91-8816224516
}

\begin{abstract}
In an application like radar there is a need for a wide range of Beam widths depending on whether the radar is operating in search mode or tracking mode. Wide range of beam widths can be achieved by using optimization algorithms like Biogeography-based optimization (BBO) and Differential Evolution Algorithm (DE). The desired beam width should be achieved without any significant increase in the side lobe level (SLL). This can be done by optimizing both SLL and FNBW simultaneously. Synthesis of linear array antenna for a fixed range of beam width is obtained by using the proposed methodology. The results for simultaneous optimization of FNBW and SLL using $\mathrm{BBO}$ and DE algorithms are compared.
\end{abstract}

Keywords: Array Factor (AF); Biogeography-based optimization (BBO); Differential Evolution Algorithm (DE); Side lobe level (SLL); First Null Beam width $(F N B W)$.

\section{Introduction}

In an application like radar, there is need for a broad beam while radar is operating in search mode and once an indication of a target is obtained, the radar has to switch over to tracking mode where a narrow main beam is required. In both cases, a low side lobe level is required to avoid false echoes. With the conventional synthesis methods trying to reduce SLL results in an increase of FNBW and vice versa. Using random stochastic methods like BBO and DE, optimization of FNBW and SLL can be done.

Trying to reduce SLL leads an increase in the first null beam width. In many applications a narrow beam width and low side lobe level beams are required but a significant decrease in FNBW leads to an increase in SLL and vice versa. In order to achieve a desired range of beam widths along with low side lobe levels, both SLL and FNBW are to be optimized at the same time, so that the best combination of these two parameters can be obtained simultaneously [3].

The main requirement here is to obtain a desired FNBW with a low side lobe level. To get a low side lobe level beam with desired value of FNBW a linear antenna array is synthesized. This can be done by optimizing both FNBW and SLL simultaneously by using $\mathrm{BBO}$ or DE techniques. Results obtained with $\mathrm{BBO}$ and $\mathrm{DE}$ algorithms are compared. Software is implemented using MATLAB R2013a.

\section{Preliminaries}

\subsection{Linear Array}

Linear array antenna consists of set of antenna elements placed along a straight line and there will be a progressive phase shift among the excitations of the elements of the array. The properties of the Linear array mainly depend upon the number of elements in the array $(\mathrm{N})$, the spacing between each element $(\mathrm{d})$, the radiation pattern of each antenna and the phase of these elements[1].

In this paper a uniform linear array is considered where the spacing between the elements is a half wave length and progressive phase shift is zero. Array factor (AF) gives the directive nature and radiation pattern of the array. The array radiation pattern is obtained by multiplication of array factor with the element pattern. The array factor AF is given by

$\mathrm{AF}=E_{0} e^{j \beta z_{0} \cos \theta}+E_{1} e^{j \beta z_{1} \cos \theta}+\ldots \ldots .+E_{N-1} e^{j \beta z_{N-1} \cos \theta}$

Where $\mathrm{E}_{0}, \mathrm{E}_{1} \ldots$ Represent the excitation amplitudes of the radiation elements and $\beta$ is given by $2 \pi / \lambda$.

\subsection{Fitness Function}

For optimization of a parameter like FNBW or SLL, a Fitness function is defined and for every set of amplitudes of the antenna Linear array, the Fitness function is evaluated and the sets of amplitudes with the best fitness value are selected. Finally the solutions giving the required value of FNBW or SLL are retained. In this paper, the objective is to obtain a desired value of FNBW with a low side lobe level. To achieve this, both FNBW and SLL are to be optimized simultaneously.

To obtain a narrow main beam with low side lobe level, the fitness function is taken as

Fitness function $=\left[\mathrm{c} 1 *\left\{\mathrm{FNBW}_{\text {desired }}-\mathrm{FNBW}_{\text {obtained }}\right\}+\right.$

$$
\left.\mathrm{c} 2 *\left\{\operatorname{abs}\left(\mathrm{SLL}_{\text {obtained }}\right)-\operatorname{abs}\left(\mathrm{SLL}_{\text {desired }}\right)\right\}\right]
$$

In the above fitness function values of $\mathrm{FNBW}_{\text {desired }}$ and $\mathrm{SLL}_{\text {desired }}$ 
are assigned according to the termination criteria. Here $\mathrm{c} 1$ and $\mathrm{c} 2$ must be selected according to the priorities of the reduction of side lobe level or first null beam width.

\section{Biogeography- Based Optimization (BBO)}

\subsection{Algorithm Definition.}

BBO is an evolutionary algorithm which is computed iteratively. It is generally used to optimize multi-dimensional valued function. This algorithm is based on the migration of species between islands and the presence of species. Islands with high habitat suitability index (HSI) are friendly to live. The Islands with high HSI will support many species which leads an increase in the emigration rate and the islands with low HSI will have high immigration rates as the species tend to leave the island [6].

Steps involved in BBO technique are selection of population, determination of immigration and emigration rates, migration and mutation operation, replacement of worst habitants, updating the best values, and checking termination criteria. These steps are repeated for certain number of iterations or until the termination criteria are met as shown in Figure 1.

Firstly a random set of elements are selected and emigration rate (ER) and immigration rate (IR) are calculated as given below.

$E R=[(\mathrm{N}+1)-(1: \mathrm{N})] /[\mathrm{N}+1]$

$\mathrm{IR}=1-\mathrm{ER}$

Mutation operation is applied after calculating the ER and IR rates where mutation is the process of changing or mutating a single value from the set of excitation amplitudes.

\subsection{Software design for $\mathrm{BBO}$.}

Algorithm

- Inputs to be given: number of elements in the array, spacing between them, desired SLL and/or FNBW value.

- Control Parameter of the array is to be chosen i.e. Emigration rate and Immigration rate parameters.

- Generation of initial population randomly

- Defining fitness function

- Finding SLL and/or BWFN of each particle of initia population.

- Determination of immigration and emigration rates.

- Performing migration operation

- Performing mutation operation

- Evaluating fitness function

- Ranking all the particles according to their fitness values and choosing the best two values as Elite costs.

- Replacing the worst habitants with elite solutions.

- Evaluating fitness function and sorting the best solutions in ascending order.

- Checking the termination criteria.

- $\quad$ Else, repeating the procedure from step 5.
- Taking the optimal solution.

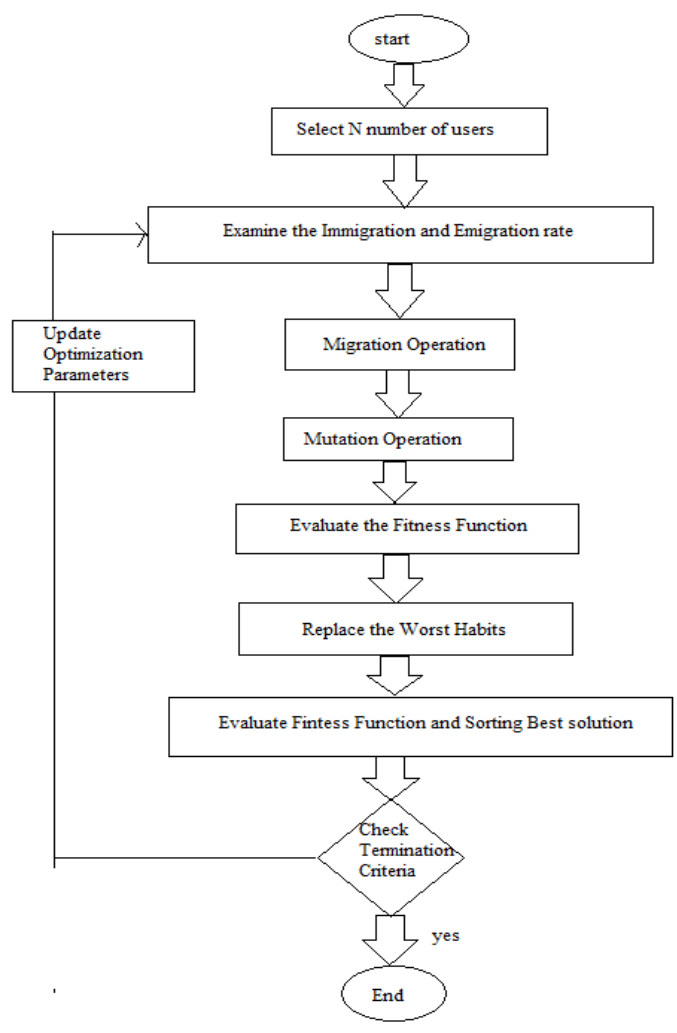

Fig. 1: Algorithm for BBO Optimization

\section{Differential Evolution Algorithm}

\subsection{Algorithm Definition.}

Differential Evolution Algorithm (DE) is similar to Genetic Algorithm (GA) as DE uses mutation operation as primary search mechanism. This algorithm is different form evolutionary algorithms as DE uses population based global search strategy along with the crossover and mutation operations. DE is preferred to GA as it reduces the complexity involved in genetic operations and the memory capacity of DE helps it to track the current search to adjust their search strategy [5]. The basic operations involved in DE algorithm are selection, crossover and mutation as shown in Figure 2.

The population of each generation $P$ contains $N$ individuals. Assuming that the individual $e$ of generation $\mathrm{P}$ is represented as

$X_{e p}=\left(X_{e p}{ }^{1}, X_{e p}{ }^{2}, \ldots \ldots X_{e p}{ }^{D}\right) \ldots \ldots$

For the Mutation Operation an individual can be generated by the following formula:

$$
X_{v 1, p+1}=X_{v 2, p+2}+E^{*}\left(X_{v 2, p^{-}} X_{v 3, p}\right) \ldots
$$

Where $\mathrm{v} 1, \mathrm{v} 2, \mathrm{v} 3$ are random numbers within $[1, \mathrm{~N}]$ and variation factor $\mathrm{E}$ is a real number. In the crossover operation the code is exchanged between the old and new generated individuals. Selection process selects the fitness values of the individuals after mutation and crossover operations with the target individuals. 


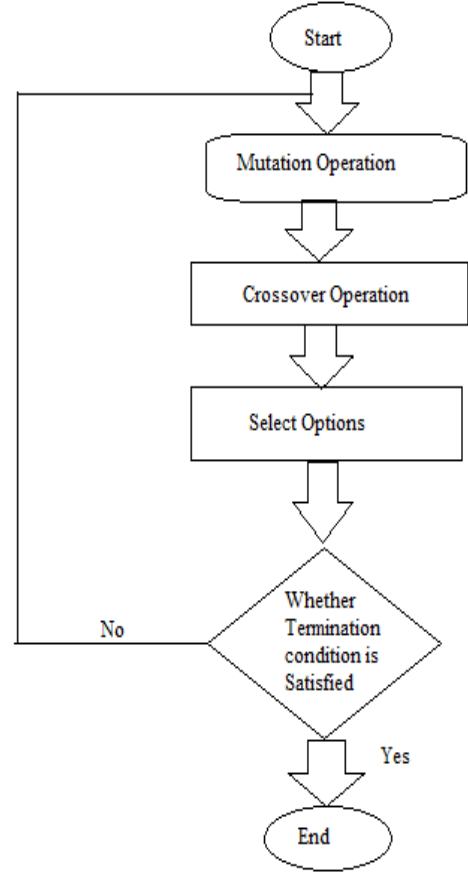

Fig. 2: Algorithm for DE Optimization

\subsection{Software Design for DE.}

Algorithm

- Inputs to be given: number of elements in the array, spacing between them, desired SLL and/or FNBW value.

- Control Parameter of the array is to be chosen i.e. crossover probability parameters.

- Generation of initial population randomly

- Defining fitness function.

- Finding SLL and/or BWFN of each combination of amplitudes of initial population.

- Performing mutation operation

- Performing crossover operation

- Performing selection operation

- $\quad$ Replacing the worst Fitness values with best values.

- Checking the termination criteria.

- $\quad$ Else, repeating the procedure from step 5.

- $\quad$ Taking the optimal solution.

\section{Proposed Methodology}

In this paper, synthesis of linear array antenna is implemented using DE to obtain a desired FNBW with a low side lobe level [9]. To synthesize the linear array, the input data to be given are the required beam width, minimum value of SLL, spacing (d) and progressive phase shift of the array.

The proposed method generates a wide range of beam widths from 5 degrees to 125 degrees without any significant increase in the side lobe level which is kept below $-18 \mathrm{~dB}$. The algorithm is shown in Figure 3.

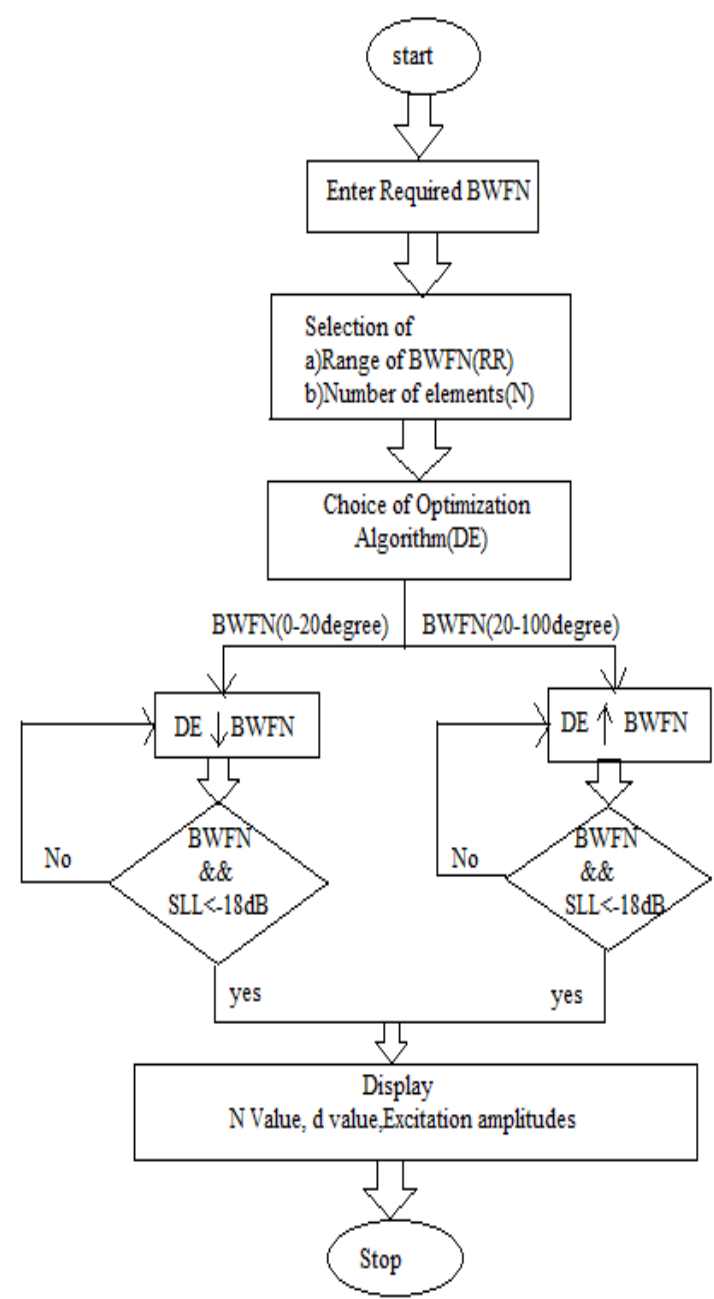

Fig. 3: Algorithm for Proposed methodology.

\subsection{Software Design for Proposed Method.}

- Inputs to be given: required beam width / beam width range, minimum value of SLL, spacing (d) and progressive phase shift.

- Selection of number of elements $(\mathrm{N})$ and range of beam width (RR)

- Choice of the Optimization algorithm (In this paper Differential Evolution Algorithm (DE) optimization is used).

- Checking whether range of beam width is above or below 20 degrees.

- Using DE for increase in FNBW if RR $>20^{\circ}$ degrees.

- Using DE for simultaneous optimization of FNBW and SLL for a decrease of FNBW without increase in SLL if $\mathrm{RR}<20^{\circ}$ degrees.

- After optimization checking for termination condition

1. If obtained FNBW is within the given range.

2. If obtained SLL $<-18 \mathrm{~dB}$

- $\quad$ Else, repeat procedure from step5.

- If termination criteria is met, display number of elements $(\mathrm{N})$ and excitation amplitudes (E).

- $\quad$ Stop. 


\section{Results and Discussion}

Implementation of these algorithms is carried out using MATLABR2013a software. The results are obtained for the optimization of First null beam width (FNBW) and side lobe level (SLL) for BBO and DE algorithms.

\subsection{Results for Uniform Linear Array Antenna.}

All the elements in the array are having uniform amplitude and uniform spacing.

\subsubsection{Values of SLL and FNBW for Uniform Linear Array.}

For uniform Linear Array all the elements are placed with uniform spacing generally placed at 0.5 lamda between each element. The amplitude for every antenna element is constant and generally the value is taken as one. The values of FNBW and SLL are obtained for different number of elements in linear array and tabulated in Table 1.

Table 1: Values of SLL and FNBW for uniform linear array

\begin{tabular}{cccc}
\hline No.of elements (N) & $\begin{array}{c}\text { Spacing (d) in } \\
\lambda\end{array}$ & $\begin{array}{c}\text { Side lobe } \\
\text { level (SLL) } \\
\text { in dB }\end{array}$ & $\begin{array}{c}\text { First Null } \\
\text { Beamwidth } \\
\text { (FNBW) } \\
\text { in degrees }\end{array}$ \\
\hline 30 & 0.5 & -13.26 & 9.20 \\
20 & 0.5 & -13.19 & 12.40 \\
16 & 0.5 & -13.14 & 16.0 \\
10 & 0.5 & -12.96 & 22.91 \\
\hline
\end{tabular}

The radiation pattern for array factor with $\mathrm{N}=30$ is shown in Figure 4.

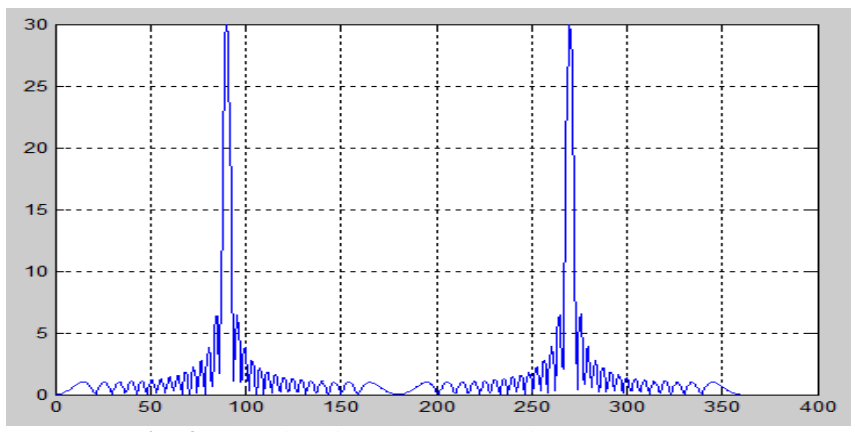

Fig. 4: Plot of uniform linear array for case $(\mathrm{N}=30)$.

\subsection{Results of BBO Optimization.}

For non uniform linear array the amplitude for excitation elements are varied randomly and the spacing between these elements is taken as $0.5 \lambda$ and progressive phase shift is taken as zero.

\subsubsection{Values of SLL/ FNBW Using BBO based Optimization.}

The values of SLL/FNBW are obtained by using BBO based optimization and values are tabulated in Table 2.

\begin{tabular}{ccccc}
\multicolumn{4}{c}{ Table 2: Values of SLL/FNBW using BBO based Optimization. } \\
\hline $\begin{array}{c}\text { No.of } \\
\text { element } \\
(\mathrm{N})\end{array}$ & $\begin{array}{c}\text { SLL (in dB) } \\
\text { Before } \\
\text { optimization }\end{array}$ & $\begin{array}{c}\text { SLL } \\
\text { (in dB) } \\
\text { Using BBO }\end{array}$ & $\begin{array}{c}\text { FNBW } \\
\text { (degree) } \\
\text { Before } \\
\text { optimization }\end{array}$ & $\begin{array}{c}\text { FNBW } \\
\text { (degree) } \\
\text { Using BBO }\end{array}$ \\
\hline 30 & -13.26 & -27.72 & 9.20 & 5.20 \\
20 & -13.19 & -25.26 & 12.40 & 7.40 \\
16 & -13.14 & -24.15 & 16.0 & 8.80 \\
10 & -12.96 & -22.32 & 22.91 & 13.80 \\
\hline
\end{tabular}

The radiation pattern for array factor with $\mathrm{N}=16$ using $\mathrm{BBO}$ optimization is shown in Figure 5.

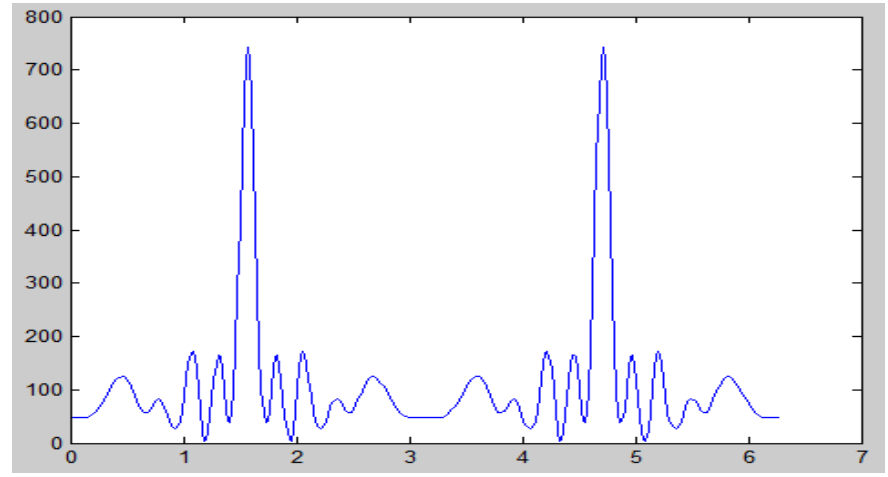

Fig. 5: Plot of Array factor using $B B O$ for case $(\mathrm{N}=16)$

6.2.2. Values of SLL and FNBW using BBO based Optimization.

The values of FNBW and SLL are obtained by using BBO based optimization of both FNBW and SLL simultaneously at once. The values are tabulated in Table $\mathbf{3}$.

Table 3: Values of SLL and FNBW using BBO based Optimization. No.of elements (N) Spacing (d) in $\lambda \quad$ BBO optimization FNBW and SLL

\begin{tabular}{llll}
\hline 30 & 0.5 & 6.4 & -21.41 \\
20 & 0.5 & 9.4 & -19.16 \\
16 & 0.5 & 12.0 & -18.83 \\
10 & 0.5 & 16.8 & -17.22 \\
\hline
\end{tabular}

\subsection{Results of DE Optimization.}

For non uniform linear array the amplitude for radiation elements are varied randomly and the spacing between these elements is kept constant to $0.5 \wedge$.

Table 4: Values of SLL/FNBW using DE based Optimization.

\begin{tabular}{ccccc}
\hline $\begin{array}{c}\text { No.of } \\
\text { element } \\
(\mathrm{N})\end{array}$ & $\begin{array}{c}\text { SLL (in dB) } \\
\text { Before } \\
\text { optimization }\end{array}$ & $\begin{array}{c}\text { SLL } \\
\text { (in dB) } \\
\text { Using DE }\end{array}$ & $\begin{array}{c}\text { FNBW } \\
\text { (degree) } \\
\text { Before } \\
\text { optimization }\end{array}$ & $\begin{array}{c}\text { FNBW } \\
\text { (degre) } \\
\text { Using DE }\end{array}$ \\
\hline 30 & -13.26 & -28.43 & 9.20 & 4.80 \\
20 & -13.19 & -27.32 & 12.40 & 6.80 \\
16 & -13.14 & -25.67 & 16.0 & 8.00 \\
10 & -12.96 & -24.89 & 22.91 & 9.40 \\
\hline
\end{tabular}

6.3.1. Values of SLL/ FNBW using DE based optimization.

The values of SLL/FNBW are obtained by using DE based optimization and values are tabulated in Table 4.

The radiation pattern for array factor with $\mathrm{N}=10$ using DE optimization is shown in Figure 6.

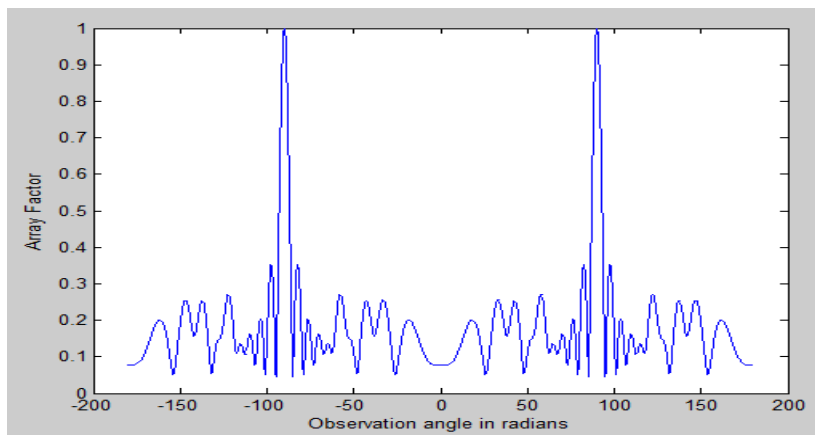

Fig. 6: Plot of Array factor using $B B O$ for case $(\mathrm{N}=16)$ 


\subsubsection{Values of SLL and FNBW using DE based optimization.}

The values of FNBW and SLL are obtained by using DE based optimization of both FNBW and SLL simultaneously at once. The values are tabulated in Table 5 .

Table 5: Values of SLL/FNBW using DE based Optimization.

\begin{tabular}{cccc}
\hline $\begin{array}{c}\text { No.of elements } \\
(\mathrm{N})\end{array}$ & $\begin{array}{c}\text { Spacing }(\mathrm{d}) \text { in } \\
\lambda\end{array}$ & \multicolumn{2}{c}{$\begin{array}{c}\text { DE optimization } \\
\text { FNBW and SLL }\end{array}$} \\
\hline 30 & 0.5 & 5.80 & -22.21 \\
20 & 0.5 & 8.80 & -20.64 \\
16 & 0.5 & 11.4 & -19.12 \\
10 & 0.5 & 15.1 & -18.35 \\
\hline
\end{tabular}

\subsection{Comparison of FNBW and SLL using BBO and DE} Optimization.

The values of SLL and FNBW are obtained from BBO and DE optimization are compared and tabulated in Table 6.

Table 6: Values of SLL and FNBW using BBO and DE based Optimization.

\begin{tabular}{ccccccc}
$\begin{array}{c}\text { No.of } \\
\text { elements } \\
(\mathrm{N})\end{array}$ & $\begin{array}{c}\text { FNBW, SLL } \\
\text { Before optimiza- } \\
\text { tion }\end{array}$ & \multicolumn{2}{c}{$\begin{array}{c}\text { FNBW, SLL } \\
\text { Using BBO }\end{array}$} & \multicolumn{2}{c}{$\begin{array}{c}\text { FNBW, SLL } \\
\text { Using DE }\end{array}$} \\
\hline 30 & 9.2 & -13.26 & 6.4 & -21.41 & 5.8 & -22.21 \\
20 & 12.4 & -13.19 & 9.4 & -19.16 & 8.8 & -20.64 \\
16 & 16.0 & -13.14 & 12.0 & -18.83 & 11.4 & -19.12 \\
10 & 22.9 & -12.96 & 16.8 & -17.22 & 15.1 & -18.35 \\
\hline
\end{tabular}

From the comparison results it is clear that DE optimization produce better results than using $\mathrm{BBO}$ optimization.

\subsection{Results of Proposed Methodology.}

The required beam width is given as input for the algorithm and the outputs are no. of elements $(\mathrm{N})$ and excitation amplitudes. The results for given inputs are tabulated in Table 7. In this paper DE algorithm is used as optimization algorithm.

Table 7: Values of SLL and FNBW obtained with Proposed Methodology.

\begin{tabular}{|c|c|c|c|c|c|c|}
\hline \multirow{2}{*}{$\begin{array}{c}\text { INPUT } \\
\begin{array}{c}\text { FNBW } \\
\text { required } \\
\text { (degree) }\end{array} \\
\end{array}$} & \multicolumn{6}{|c|}{ OUTPUT } \\
\hline & $\begin{array}{l}\text { No. of } \\
\text { elements } \\
(\mathrm{N})\end{array}$ & $\begin{array}{c}\text { FNBW } \\
\text { Obtained } \\
\text { (degree) }\end{array}$ & $\begin{array}{l}\text { SLL } \\
\text { (dB) }\end{array}$ & \multicolumn{3}{|c|}{ Excitation amplitudes } \\
\hline & 16 & 99 & - & 0.2659 & 0.1319 & 0.5303 \\
\hline & & & 23.58 & 0.4456 & 0.8382 & 0.3971 \\
\hline & & & & 5.0000 & 4.1407 & 2.0043 \\
\hline & & & & 1.5783 & 0 & 0.2813 \\
\hline & & & & & 0.2131 & 0.2444 \\
\hline & & & & 0.3746 & & \\
\hline \multirow[t]{6}{*}{$90-80$} & 16 & 86 & - & 0.2342 & 0.0058 & 2.6290 \\
\hline & & & 21.32 & 1.5489 & 4.3942 & 4.6006 \\
\hline & & & & 5.0000 & 3.6835 & 0.1159 \\
\hline & & & & 0.5873 & 0.6056 & 0.6025 \\
\hline & & & & 1.0028 & 1.2758 & 0.2775 \\
\hline & & & & 0.6076 & & \\
\hline \multirow[t]{7}{*}{$80-70$} & 20 & 75 & - & 0.8938 & 0.0541 & 1.8428 \\
\hline & & & 21.18 & 0.0738 & 2.0347 & 0.9774 \\
\hline & & & & 0.7282 & 0.7294 & 3.3436 \\
\hline & & & & 2.6990 & 3.8808 & 5.0000 \\
\hline & & & & 4.8023 & 3.2811 & 3.9709 \\
\hline & & & & 1.1947 & 0.5928 & 1.68140 \\
\hline & & & & 0 & & \\
\hline \multirow[t]{7}{*}{$70-60$} & 20 & 62 & - & 0 & $\begin{array}{ll}0 & 0.0767\end{array}$ & 0.6446 \\
\hline & & & 20.92 & 0 & 1.6450 & 1.3560 \\
\hline & & & & 2.4623 & 2.0919 & 3.6091 \\
\hline & & & & 1.7931 & 5.0000 & 5.0000 \\
\hline & & & & 5.0000 & 5.0000 & 2.4018 \\
\hline & & & & 2.8568 & 1.8047 & 0.4022 \\
\hline & & & & 0.6605 & & \\
\hline \multirow[t]{2}{*}{$60-50$} & 25 & 53 & - & 3.5321 & 0.1338 & 0 \\
\hline & & & 20.56 & $\begin{array}{ll}0 & 0.8\end{array}$ & $\begin{array}{ll}045 & 2.467\end{array}$ & 6 \\
\hline
\end{tabular}

\begin{tabular}{|c|c|c|c|c|c|c|}
\hline & & & & \multicolumn{2}{|c|}{4.0134} & \multirow{2}{*}{$\begin{array}{l}2.6815 \\
4.2693\end{array}$} \\
\hline & & & & 1.2537 & 1.7565 & \\
\hline & & & & 4.7240 & 4.3389 & 4.9361 \\
\hline & & & & 3.5173 & 3.0210 & 2.9127 \\
\hline & & & & 0.9224 & 0.8154 & 0 \\
\hline & & & & 0.6130 & 2.4590 & 0.8766 \\
\hline \multirow[t]{8}{*}{$50-40$} & 27 & 47 & - & 1.2905 & 0 & 0.8192 \\
\hline & & & 21.32 & 1.2240 & 0.8513 & 1.1137 \\
\hline & & & & 0.1316 & 0 & 1.6445 \\
\hline & & & & 0.1709 & 1.6673 & 0 \\
\hline & & & & 0.9527 & 5.0000 & 5.0000 \\
\hline & & & & 4.2005 & 5.0000 & 5.0000 \\
\hline & & & & 3.9748 & 0.5632 & 3.6502 \\
\hline & & & & $\begin{array}{rr}0 & 4.8912 \\
0 & 2.035\end{array}$ & 570.1035 & 0.4506 \\
\hline \multirow[t]{3}{*}{$20-10$} & 10 & 17 & 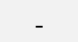 & 5.0000 & $\begin{array}{ll}0 & 0\end{array}$ & 0.1455 \\
\hline & & & 18.87 & 0.2426 & 0 & 00.273 \\
\hline & & & & $\begin{array}{ll}0 & 4.4541\end{array}$ & & \\
\hline \multirow{5}{*}{$10-5$} & 16 & 9 & - & 4.9793 & 2.2267 & 2.4843 \\
\hline & & & 19.92 & $0 \quad 0$ & 0.4978 & 0.0256 \\
\hline & & & & 0.3496 & 0 & 0.2346 \\
\hline & & & & 0.1960 & 0 & 0.0313 \\
\hline & & & & $\begin{array}{ll}0 & 3.8928\end{array}$ & $28 \quad 5.0000$ & \\
\hline
\end{tabular}

\section{Conclusions}

In this paper, linear antenna array is synthesized to obtain the desired range of beam width with low side lobe level using BBO and DE optimization techniques. The best results are obtained with DE technique compare to $\mathrm{BBO}$. The range of beam widths obtained with DE technique is from 5.8 degrees to 99 degrees and the SLL corresponding to 5.8 degrees is $-22.21 \mathrm{~dB}$ and the SLL corresponding to 99 degrees is $-23.58 \mathrm{~dB}$. It is observed that the SLL is increasing only slightly as the beam width is decreased from 99 degrees to 5.8 degrees.

\section{References}

[1] IqroopKaur, Sanjeev Kumar "linear antenna array optimization techniques", International Journal of Engineering Trends and Technology(2011), Available online: http://www.ijettjournal.org/volume-1/issue1/ijettjournal-v1i1p9.pdf

[2] BiulGoswami, DurbadalMandal, "A genetic algorithm for level control of nulls and side lobes in linear antenna arrays", Journal of king sand university- computer and information sciences. Available online: https://www.sciencedirect.com/science/article/pii/S1319157812000328

[3] R. Storn, " On the usage of differential evolution for function optimization", 1996 Biennial Conference of the North American, (1996), 519 523. Available online: http://www.icmlc.org/icmlc2009/011_icmlc2009.pdf

[4] L. D. S. Coelho, V. C. Mariani, "Combining of chaotic differential evolution and quadratic programming for economic dispatch optimization with valve-point effect", Power Systems IEEE Transactions on, 21, (2006), 989-996. Available online: https://www.hindawi.com/journals/aaa/2014/175417/ref/

[5] C.-T. Su, C.-S. Lee, "Network reconfiguration of distribution systems using improved mixed-integer hybrid differential evolution", Power Delivery IEEE Transactions on, 18, (2003), 1022-1027. Available online: http://ieeexplore.ieee.org/iel5/61/27186/01208393.pdf

[6] M. Mussetta, P. Pirinoli, R. E. Zich, "Ap-plication of modified BBO to microstrip filter optimization", 2013 IEEE Antennas and Propagation Society International Symposium (AP-SURSI), (2013), 410-411. Available online: http://ieeexplore.ieee.org/document/6710866/

[7] W.-C. Weng, F. Yang, A. Z. Elsherbeni, "Linear Antenna Array Snythesis Using Taguchi's Method: A Novel optimization Technique in Electromagnetics", IEEE Transactions on Antennas and Propagation, 55, 3, (2007) 723-730. Available online: http://ieeexplore.ieee.org/document/4120302/

[8] M. M. Khodier, C. G. Christodoulou, "Linear Array Geometry Synthesis With Minimum Sidelobe Level and Null Control Using Particle Swarm Optimization", IEEE Transactions on Antennas and Propagation, 53, 8, (2005) 2674-2679. Available online: http://ieeexplore.ieee.org/document/1492619/

[9] T.prakash, V.P. Singh, D.P.S. Chauhan "Computational Intelligence \& Communication Technology (CICT), 2016 Second International Conference", IEEE DOI:10.1109/CICT.2016.60. Available online http://ieeexplore.ieee.org/abstract/document/7546614/?section=abstract 